\title{
Interés situacional en clase de Lengua Española en secundaria: relaciones estructurales con el compromiso, el desapego y el rendimiento*
}

\author{
Situational Interest in Spanish Language Class on Secondary Education: \\ Structural Relations with Engagement, Disaffection, and Performance
}

Recibido: mayo 10 de 2012 | Revisado: octubre 15 de 2012 | Aceptado: octubre 22 de 2012

\author{
ANTONIO GONZÁLEZ FeRNÁNDEZ ** \\ Universidad de Vigo, Pontevedra, España \\ PaOla Verónica PAOLONi*** \\ MaRÍA CRISTINA RINAUdo \\ DANILO DONOLO
}

Universidad Nacional de Río Cuarto, Córdoba, Argentina

doi:10.11144/Javeriana.UPSY12-3.iscl

Para citar este artículo: González, A., Paoloni, V., Rinaudo, M. C. \& Donolo, D. (2013). Interés situacional en clase de Lengua Española en secundaria: relaciones estructurales con el compromiso, el desapego y el rendimiento. Universitas Psychologica, 12(3), 753-765. doi:10.11144/Javeriana.UPSY12-3. iscl

* Este estudio forma parte de una investigación coordinada por el Dr. González Fernández y realizada en el marco de estudios comparados entre España, Portugal, Brasil y Argentina sobre aspectos motivacionales y emocionales implicados en los aprendizajes y rendimiento académico de estudiantes del nivel medio.

** Universidad de Vigo, Pontevedra, España. Profesor Titular del Área de Psicología Básica. Imparte docencia en Psicopedagogía, Trabajo Social y Educación Social en la Facultad de Ciencias de la Educación E-mail: aglez@uvigo.es

**** Universidad Nacional de Río Cuarto, Córdoba, Argentina. La Dra. Paoloni y el Dr. Donolo son investigadores del Consejo Nacional de Investigaciones Científicas y Técnicas de la República Argentina. Al igual que la Dra. Rinaudo, se desempeñan como docentes en Carreras de Educación en la Universidad Nacional de Río Cuarto, Argentina. E-mails: paopaoloni17@hotmail.com, crinaudo@hum.unrc. edu.ar, donolo@hum.unrc.edu.ar
RESUMEN

El objetivo de este estudio fue evaluar un modelo que relaciona interés, compromiso y rendimiento. El estudio evaluó tres modalidades de interés situacional (activado, mantenido-afecto y mantenido-valor), el compromiso conductual, el desapego conductual y el rendimiento en clase de Lengua Española. Estas variables se evaluaron en el aula a lo largo de un período de nueve meses en una muestra de 565 alumnos españoles de Educación Secundaria Obligatoria (ESO), con edades comprendidas entre 12 y 17 años $(M=13.78$ años; $D E=1.14)$. Una serie de modelos de ecuaciones estructurales reveló que las tres modalidades del interés situacional predijeron significativamente el compromiso, el desapego y el rendimiento; compromiso y desapego predijeron el rendimiento; como se había hipotetizado, compromiso y desapego mediaron los efectos del interés situacional sobre el rendimiento académico. Para concluir, se discuten los resultados y sus implicaciones para la teoría del interés y para los programas de intervención educativa.

Palabras clave autores

Compromiso conductual, desapego, interés situacional, Lengua Española, rendimiento académico.

Palabras clave descriptores

Psicología educativa, aprendizaje, aspectos motivacionales, emocionales y cognitivos.

\section{A B S T R A C T}

The purpose of this study was to test a model linking interest, engagement, and performance. The study assessed three types of situational interest (triggered, maintained-feeling, and maintained-value), behavioral engagement, behavioral disaffection, and performance in Spanish language classes. These variables were assessed in the classroom over a nine-month period on a sample of 565 Spanish secondary students aged 12 to 17 years $(\mathrm{M}$ age $=$ 13.78 years; $S D=1.14)$. A series of structural equation models revealed that the three types of situational interest significantly predicted engagement, disaffection, and performance; engagement and disaffection also predicted performance; as hypothesized, engagement and disaffection mediated the effects of situational interest on academic performance. To conclude, the implications of these findings are discussed in the light of current theory of interest and educational intervention programmes. 
Key words authors

Behavioral engagement, disaffection, situational interest, Spanish language, academic performance.

Key words plus

Educational Psychology, learning, motivational emotional and cognitive aspects.

\section{Introducción}

El rendimiento académico de adolescentes y jóvenes depende de múltiples factores personales y sociales (Caso-Niebla \& Hernández-Guzmán, 2007). Entre las variables personales que condicionan ese rendimiento destacan las motivacionales (Núñez, Martín-Albo, Paredes, Rodríguez \& Chipana, 2011; Valle et al., 2010), las emocionales (Feldman et al., 2008; Macías-Martínez \& Hernández-Pozo, 2008; Valle et al., 2010) y las estratégicas (Caso-Niebla \& Hernández-Guzmán, 2007; Pérez, Valenzuela, Díaz, González-Pienda \& Núñez, 2011).

Muy próximo a estos tres factores - motivación, emoción y estrategias- se encuentra el engagement académico, un término traducido al castellano como vinculación psicológica con los estudios (Salanova, Martínez, Bresó, Llorens \& Grau, 2005), implicación con la escuela (González González, 2010; Ros, 2009) o compromiso académico (Extremera, Durán \& Rey, 2007; González González, 2010; Manzano, 2004; Parra, 2010). En el contexto educativo también se ha analizado su opuesto, que se operativiza como burnout (Caballero, Abello \& Palacio, 2007; Caballero, Hederich \& Palacio, 2010; Extremera et al., 2007; Manzano, 2004) o como disaffection, un término que se ha traducido por desapego, desafección, falta de implicación, desenganche, deserción o abandono de los estudios (García, Casal, Merino \& Sánchez, 2012; González González, 2010; Martínez-Arias, Aguado, Álvarez, Colodrón \& Gallego, 2007).

\section{Interés personal y situacional}

Si algún concepto motivacional puede considerarse definitorio de la identidad propia de un estudiante, ese es su interés por un tema o una materia (Renninger, 2009). La importancia del interés queda subrayada en el último informe PISA para España (Ministerio de Educación, 2010), donde se asegura que el interés de un alumno por una asignatura es una garantía de éxito en la misma, mientras que su desinterés por esta preludia un rendimiento muy limitado. El interés suele caracterizarse como un fenómeno que emerge a partir de la interacción entre un individuo y su entorno (Hidi \& Renninger, 2006; Hidi, Renninger \& Krapp, 2004). Por esto, suelen diferenciarse dos tipos de interés, el personal y el situacional.

El interés personal o individual se concibe como una preferencia personal relativamente duradera por ciertos temas o actividades (Schiefele, 2009); está basado en el conocimiento y la valoración de una clase de objetos o ideas y suscita en el alumno el deseo de implicarse en actividades relacionadas con el tema. Hidi et al. (2004), Krapp y Prenzel (2011) y Schiefele (2009) identifican varios componentes del interés personal: el primero incluye una serie de sentimientos asociados a la interacción con un objeto de interés, como disfrute, activación e implicación; y el segundo, la valoración, se refiere a la atribución de una especial importancia o relevancia personal a un objeto o tema.

En cuanto al interés situacional o contextual en el ámbito educativo, Hidi y Renninger (2006) afirman que es evocado por algún elemento del entorno más próximo, por lo que puede mantenerse durante largo tiempo, influyendo en el interés personal, o puede desaparecer de forma inmediata. En este sentido, Hidi y Renninger (2006), Hidi et al. (2004), Krapp y Prenzel (2011) y Renninger (2009) diferencian dos modalidades de interés situacional, el activado y el mantenido. El interés situacional activado capta la atención del sujeto, despertando en él diversas experiencias afectivas relacionadas con el entorno; en algunos casos puede ser el inicio de un interés personal. Por su parte, el interés situacional mantenido demanda mayor implicación del sujeto, pues este comienza a establecer una conexión más significativa con un determinado contenido al descubrir su importancia y utilidad.

A pesar de que son distintos conceptualmente, el interés situacional puede activar y mantener el personal (Hidi \& Renninger, 2006; Hidi et al., 
2004; Krapp \& Prenzel, 2011; Renninger, 2009). El interés situacional activado inicia este proceso llamando la atención del alumno hacia un determinado tema, aunque esta experiencia no se traduce necesariamente en un interés personal. Es el interés situacional mantenido el que proporciona ese nexo: una vez atraída la atención de los estudiantes hacia un tema, es probable que aquellos que lo consideran atractivo e importante lo valoren más y que busquen nuevas oportunidades para entrar en contacto con él, lo que dará lugar al inicio del interés personal.

El interés situacional y sobre todo el personal se relacionan positivamente con el rendimiento en diferentes niveles educativos y materias (Ainley, 2012; Alonso Tapia, 2007; Durik \& Harackiewicz, 2007; García-Señorán \& González González, 2008; Gutiérrez \& López, 2012; Hulleman, Godes, Hendricks \& Harackiewicz, 2010; Schiefele, 2009; Zhu et al., 2009).

\section{Compromiso académico vs. desapego}

El análisis del compromiso académico se ha abordado desde diferentes perspectivas (Fredricks, Blumenfeld \& Paris, 2004; Martin, 2008; Skinner \& Pitzer, 2012; Wang \& Holcombe, 2010). Estos autores han diferenciado tres componentes del compromiso que ya se consideran clásicos: el conductual, como el esfuerzo; el emocional, como el entusiasmo y el cognitivo, como las estrategias de aprendizaje.

En el contexto educativo, el compromiso conductual se define como aquellas interacciones del alumno con su entorno académico que son activas, dirigidas a metas, flexibles, constructivas y persistentes (Fredricks et al., 2004; Martin, 2008; Skinner, Kinderman \& Furrer, 2009). Algunos indicadores del compromiso conductual son el esfuerzo, la atención, la concentración o la persistencia. En general, estas conductas van acompañadas de emociones como entusiasmo, disfrute, satisfacción, orgullo y vitalidad. Este compromiso conductual suele producirse juntamente con otro tipo de compromiso, el cognitivo, que se concreta en la utilización de estrategias y en la autorregulación del aprendizaje (Cleary \& Zimmerman, 2012; Wolters \& Taylor, 2012).
En el extremo opuesto al compromiso encontramos el desapego conductual que se operativiza como ausencia de esfuerzo, distracción, escasa persistencia y aplazamientos en la realización de tareas (Martin, 2008; Skinner \& Pitzer, 2012). Este constructo estaría próximo a los de burnout, pasividad, indiferencia, desmotivación o indefensión. Habitualmente, estos comportamientos van acompañados de emociones como resignación, desaliento, tristeza, apatía, aburrimiento o frustración, sentimientos que en determinadas situaciones pueden desembocar en el estrés académico (Barraza, 2008; Extremera et al., 2007; Feldman et al., 2008), un condicionante decisivo de la salud mental de los estudiantes. De acuerdo con Skinner, Kinderman, Connell y Wellborn (2009), es necesario un análisis exhaustivo de la falta de implicación y el desapego en aquellos contextos de los que no pueden salir los sujetos, como es la escuela durante la educación secundaria obligatoria.

Numerosas investigaciones asociaron positivamente niveles elevados de compromiso con un adecuado rendimiento académico (Ainley \& Ainley, 2011; Durik \& Harackiewicz, 2007; Greene, Miller, Crowson, Duke \& Akey, 2004; Jang, 2008; Parra, 2010) y con otras variables que lo potencian (Extremera et al., 2007; González González, 2010; Manzano, 2004; Parra, 2010; Ros, 2009; Salanova et al., 2005; Skinner, Kinderman \& Furrer, 2009). Por el contrario, diferentes índices de desapego y de burnout correlacionaron negativamente con los resultados académicos (Caballero et al., 2007; Caballero et al., 2010; Martin \& Liem, 2010; Skinner, Kinderman, Connell et al., 2009) y positivamente con el fracaso escolar, el absentismo y el abandono de la escuela (García et al., 2012; González González, 2010; Martínez-Arias et al., 2007).

\section{Interés situacional y compromiso académico}

Algunos autores han constatado que el interés situacional se asoció positivamente a la persistencia, el esfuerzo y el compromiso (Ainley, 2012; Ainley \& Ainley, 2011; Durik \& Harackiewicz, 2007; Jang, 2008; Rotgans \& Schmidt, 2011; Yeung, Lau \& Nie, 2011) y negativamente al desapego (Yeung et 
al., 2011). Otros estudios recientes, aplicando modelos de ecuaciones estructurales, constataron que la atención, el esfuerzo y la persistencia actuaron como mediadores entre las estrategias utilizadas por los universitarios mientras estudiaban textos poco interesantes y el aprendizaje de conceptos (Jang, 2008); también con universitarios, la participación en clase o el trabajo en equipo mediaron las relaciones entre interés situacional y rendimiento (Rotgans \& Schmidt, 2011).

Por su parte, el esfuerzo en la realización de las tareas medió los efectos del autoconcepto y las metas de logro sobre el rendimiento en Matemáticas y Lengua Española en alumnos de secundaria obligatoria (Miñano, Gilar \& Castejón, 2012); asimismo, la vinculación psicológica con los estudios también medió los relaciones entre creencias de eficacia y nota media en universitarios (Salanova et al., 2005).

\section{Objetivos de investigación e hipótesis}

Tal como aseguran Linnenbrink-García et al. (2010), de las escalas que evalúan el interés situacional, pocas diferencian sus modalidades y componentes, por lo que no abundan investigaciones previas que los hayan analizado. Además, como reconoce Ainley (2012), apenas existen trabajos que hayan abordado la relación del interés con el compromiso conductual y el desapego en el ámbito educativo. Menos todavía son las investigaciones que los han evaluado en educación secundaria obligatoria, una etapa en la que muchos estudiantes desean abandonar el sistema educativo a pesar de que (por su edad) están obligados a continuar en él.

Por otra parte, Ainley y Ainley (2011) formulan un modelo teórico de relaciones en el que el interés situacional del alumno condiciona su nivel de compromiso, y este a su vez influye sobre su rendimiento académico. De manera análoga, Skinner, Kinderman, Connell et al. (2009) proponen que el valor de la tarea y su relevancia para el alumno condicionan sus niveles de compromiso y desapego, y que estos inciden sobre los resultados académicos logrados. Ambas propuestas pueden considerarse próximas a otros modelos, como el 3P de Biggs (Biggs, Kembler \& Leung, 2001; Justicia, Pichardo,
Cano, Berbén \& De la Fuente, 2008; Rosário et al., 2005), en los que se analiza explícitamente el papel que desempeñan ciertas variables como mediadoras (proceso) entre los predictores (presagio) y los resultados (producto).

En este contexto, el presente estudio intenta esclarecer las relaciones entre rendimiento académico, interés situacional (con sus dos modalidades, activado y mantenido, y sus dos componentes, afecto y valor), compromiso conductual y desapego, en una muestra de alumnos de educación secundaria obligatoria. A partir de estos modelos teóricos y de la investigación empírica previamente revisada, se espera que: (a) las distintas modalidades de interés situacional predigan positivamente el compromiso conductual y el rendimiento académico, y negativamente el desapego conductual; (b) el compromiso (positivamente) y el desapego (negativamente) serán predictores del rendimiento y (c) compromiso y desapego mediarán los efectos ejercidos por los tres tipos de interés situacional sobre el rendimiento académico.

\section{Método}

\section{Participantes}

Formaron la muestra 565 estudiantes con edades comprendidas entre 12 y 17 años $(M=13.78$; $D E=1.14)$, que cursaban educación secundaria obligatoria (ESO), en diferentes centros públicos y privados del noroeste de España, con una proporción equivalente de chicos y chicas. Los porcentajes de estudiantes fueron similares en los cuatro cursos, oscilando entre el $26.5 \%(N=150)$ de primero de ESO (7..$^{\circ}$ curso $)$ y el $23.2 \%(N=131)$ de cuarto de ESO (10. curso).

\section{Instrumentos}

\section{Interés situacional}

Para evaluarlo, se aplicó el Cuestionario de Interés Situacional (Linnenbrink-García et al., 2010). Esta escala está compuesta por doce ítems y evalúa tres factores, cada uno con cuatro ítems. La subescala 
de Interés situacional Activado $(\alpha=0.82)$ indaga sobre distintos aspectos de la clase que atraen la atención y despiertan el interés del estudiante (ej.: "Este curso, las clases de Lengua Española son tan entretenidas que es muy fácil estar atento/a"). La subescala de Interés situacional mantenido-Afecto $(\alpha=0.83)$ evalúa diferentes sentimientos y afectos del alumno que prolongan y sostienen su interés por esa materia (ej., "Me gustan las cosas que aprendo este año en las clases de Lengua Española"). La subescala de Interés situacional mantenido-Valor $(\alpha=0.86)$ solicita la opinión de los estudiantes sobre la relevancia, la utilidad y la valoración de lo que aprenden en clase (ej., "Las cosas que estudio en clase de Lengua Española este año son importantes para mí”). En cada ítem, los alumnos eligieron un valor entre 1 (nada verdadero para mí) y 5 (totalmente verdadero para mí). Los resultados del análisis factorial confirmatorio de esta escala indicaron que el ajuste del modelo de tres factores fue adecuado: $\chi^{2}(g l=51, N=565)=115.6, p<0.001 ; \chi^{2} / g l=$ 2.26; CFI $=0.98 ;$ RMSEA $=0.047$.

\section{Compromiso y desapego}

Para evaluarlos, se utilizó el Cuestionario de Compromiso Conductual y Desapego: versión del alumno (Skinner et al., 2008). Este instrumento incluye diez ítems agrupados en dos subescalas. La de Compromiso Conductual está compuesta por cinco ítems ( $\alpha=0.76)$ que evalúan la atención, el esfuerzo y la persistencia de los estudiantes mientras participan en las actividades de aprendizaje en el aula. (ej., "Cuando estoy en clase de Lengua Española, participo en las discusiones de clase" o "En clase de Lengua, trabajo todo lo que puedo"). La subescala de Desapego Conductual la componen cinco ítems ( $\alpha=0.82)$ que evalúan la presencia de conductas indicadoras de un escaso compromiso con el aprendizaje (ej., "Cuando estoy en clase de Lengua, hago como que trabajo" o "Cuando estoy en clase de Lengua, pienso en otras cosas"). En ambas subescalas, los alumnos estimaron su conducta eligiendo un valor entre 1 (nada verdadero para mí) y 4 (totalmente verdadero para mí). Un análisis factorial confirmatorio indicó que el modelo de dos factores se ajustó adecuadamente a los datos: $\chi^{2}$ $(g l=34, N=565)=49.5, p<0.05 ; \chi^{2} / g l=1.79 ;$ $\mathrm{CFI}=0.99 ; \mathrm{RMSEA}=0.028$.

Las versiones españolas de los dos cuestionarios (Interés y Compromiso) se elaboraron de acuerdo con el procedimiento habitual de traducción transcultural de escalas (Brislin, 1986).

\section{Rendimiento académico}

El profesor tutor de cada alumno comunicó la nota final en Lengua obtenida por cada estudiante. Las puntuaciones oscilaron entre 1 (muy deficiente) y 10 (excelente), situándose el aprobado en un valor $>5$.

\section{Procedimiento}

Las pruebas de evaluación se administraron en tres momentos diferentes: en el mes de octubre los alumnos contestaron al Cuestionario de Interés Situacional; en el mes de febrero siguiente, cumplimentaron el Cuestionario de Compromiso y Desapego y a finales de junio, los profesores comunicaron la nota final en la asignatura de Lengua. Antes de la aplicación de los cuestionarios, se solicitaron los correspondientes permisos a la dirección de los centros educativos y a las familias. Los alumnos cumplimentaron las escalas en el aula dentro del horario de docencia y ninguno se negó a contestarlas. Su aplicación en cada clase fue realizada por alguno de sus profesores. Previamente, estos les explicaron el objetivo de la investigación y la conveniencia de sinceridad en las respuestas, ofreciéndoles garantías de anonimato y confidencialidad de las mismas. Para ello, se proporcionó a cada alumno un código que mantuvo e incorporó a cada cuestionario en los diferentes momentos de la evaluación.

\section{Análisis estadísticos}

Inicialmente, se calcularon los coeficientes de fiabilidad, los estadísticos descriptivos y las correlaciones entre variables utilizando el paquete estadístico SPSS.15. Luego, se realizó un análisis factorial confirmatorio de las escalas para corroborar la idoneidad del modelo de medida, utilizando 
el programa AMOS.7. Finalmente, se realizó un análisis de ecuaciones estructurales. Como índices de ajuste se presentan los siguientes (Byrne, 2010; Hu \& Bentler, 1999): los indicadores $\chi^{2}$ y $\chi^{2} / g l$, los índices de bondad de ajuste corregido (AGFI) y de ajuste comparado (CFI), el error cuadrático medio de aproximación (RMSEA) y la raíz del residuo estandarizado cuadrático medio (SRMR).

\section{Resultados}

\section{Análisis descriptivos y correlacionales}

En la Tabla 1 aparecen las correlaciones, medias y desviaciones típicas de las variables evaluadas en este estudio. Se encontraron correlaciones positivas entre todas las variables, con la excepción del desapego que correlacionó negativamente con las demás. Los coeficientes de correlación fueron moderados, con valores entre $|0.4|$ y $|0.54|$.

\section{Modelo de medida}

Con el fin de corroborar la idoneidad de los instrumentos de evaluación aplicados, se puso a prueba el modelo de medida mediante un análisis factorial confirmatorio. Como variables latentes se consideraron las tres modalidades de interés situacional, el compromiso conductual y el desapego; como indicadores, las respuestas a los cuatro ítems que conforman cada escala de interés situacional y los cinco ítems de compromiso y desapego.

En todos los indicadores se obtuvieron índices de asimetría y curtosis menores que |1.96|, por lo que puede asumirse la normalidad univariada (Arbuckle, 2006; Byrne, 2010). No se encontraron observaciones atípicas multivariadas (outliers). Sin embargo, el coeficiente de curtosis multivariada de Mardia (20.1) superó el valor crítico (critical ratio, c.r. = 8.1). Para verificar si esta no-normalidad de los datos influía en los distintos estimadores, se realizaron dos tipos de análisis (Arbuckle, 2006; Byrne, 2010): uno con la muestra original, mediante el método de máxima verosimilitud; otro con 500 muestras aleatorias obtenidas a partir de la original por el procedimiento de remuestreo (bootstrap), también mediante el método de máxima verosimilitud; asimismo, se marcó un intervalo del 95 \% para evaluar las diferencias entre ambos análisis. No se encontraron diferencias entre los dos análisis, por lo que a continuación se presentarán los resultados obtenidos en el análisis de la muestra original (Byrne, 2010). No se efectuaron re-especificaciones del modelo de medida inicial.

Los indicadores revelan un adecuado ajuste del modelo a los datos: $\chi^{2}(g l=199, N=565)=292$, $p<0.001 ; \chi^{2} / g l=1.47$; AGFI $=0.94 ; \mathrm{CFI}=0.98$; $\mathrm{RMSEA}=0.029 ; \mathrm{SRMR}=0.033$. Además, todas las cargas factoriales estandarizadas fueron significativas $(\mathrm{b}>54 ; p<0.01)$.

\section{TABLA 1}

Estadísticos descriptivos y correlaciones entre variables

\begin{tabular}{lcccccc}
\hline \multicolumn{1}{c}{ Variables } & 1 & 2 & 3 & 4 & 5 & 6 \\
\hline 1. I.S. Activado & - & & & & & \\
2. I.S. Mantenido-Afecto & 0.47 & - & & & & \\
3. I.S. Mantenido-Valor & 0.45 & 0.49 & - & & & \\
4. Compromiso conductual & 0.41 & 0.48 & 0.49 & - & & \\
5. Desapego conductual & -0.4 & -0.44 & -0.5 & -0.43 & - & \\
6. Rendimiento académico & 0.43 & 0.47 & 0.54 & 0.52 & -0.5 & - \\
\hline Media & 3.17 & 3.36 & 3.21 & 2.64 & 2.17 & 6.66 \\
DT & 0.95 & 0.96 & 1.04 & 0.7 & 0.67 & 1.76 \\
\hline
\end{tabular}

Nota. I.S. = Interés Situacional. Todas las correlaciones fueron significativas $(p<0.01)$.

Fuente: elaboración propia. 


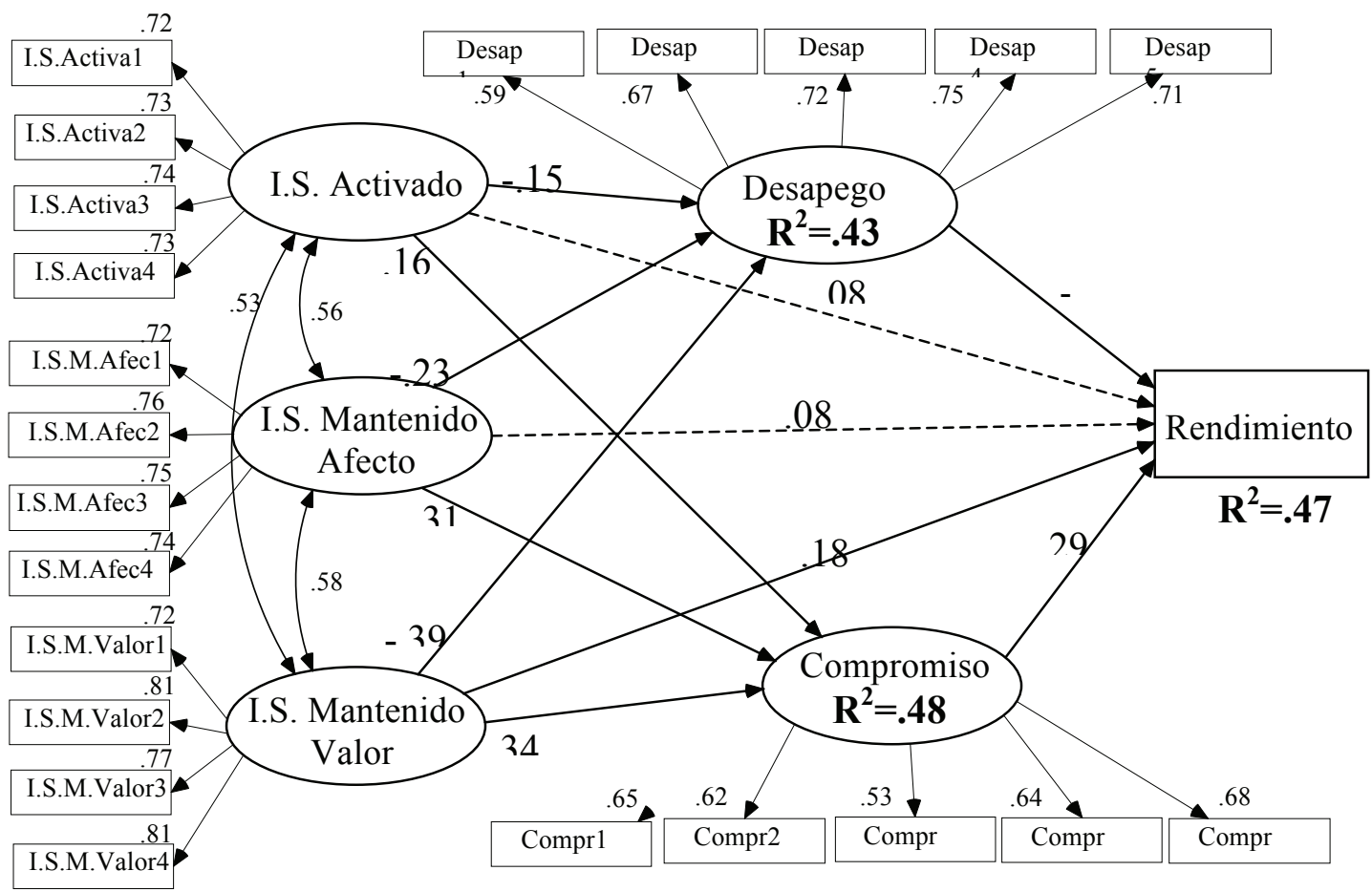

Figura 1. Modelo de relaciones estructurales entre variables.

Nota. I.S. = Interés Situacional. Los valores representan coeficientes de regresión estandarizados. Las líneas discontinuas indican relaciones no significativas $(p>0.10)$; todos los demás valores son significativos $(p<0.01)$.

Fuente: elaboración propia.

\section{Modelo estructural}

A continuación, se realizaron diferentes análisis de ecuaciones estructurales para verificar el modelo mediacional, siguiendo la propuesta de cuatro fases enumeradas por Wu y Zumbo (2008). También aquí el coeficiente de curtosis multivariada de Mardia (22.22) superó el valor crítico (c.r. $=7.78)$ en el modelo final, por lo que se llevaron a cabo los dos análisis aplicados anteriormente. Puesto que no se encontraron diferencias entre ambos análisis, se presentan los estimadores hallados en el análisis de la muestra original. La Figura 1 presenta los resultados del modelo estructural.

En una primera fase, se analizó la relación entre las modalidades de interés y el rendimiento, obteniendo un adecuado ajuste del modelo a los datos, $\chi^{2}(g l=60, N=565)=129.4, p<0.001 ; \chi^{2} / g l=$ 2.15; $\mathrm{AGFI}=0.95 ; \mathrm{CFI}=0.98$; $\mathrm{RMSEA}=0.045$; $\mathrm{SRMR}=0.032$.
En una segunda fase, se examinaron los nexos entre las modalidades de interés y los mediadores, compromiso y desapego. Los índices obtenidos revelan un adecuado ajuste del modelo a los datos, $\chi^{2}(g l=200, N=565)=299.4, p<0.001 ; \chi^{2} / g l=$ $1.49 ; \mathrm{AGFI}=0.94 ; \mathrm{CFI}=0.98 ; \mathrm{RMSEA}=0.030$; $\mathrm{SRMR}=0.034$.

El modelo mediacional total (fase 3) mostró buenos niveles de ajuste a los datos, $\chi^{2}(g l=217$, $N=565)=315.8, p<0.001 ; \chi^{2} / g l=1.45 ;$ AGFI $=0.94 ; \mathrm{CFI}=0.98 ; \mathrm{RMSEA}=0.028 ; \mathrm{SRMR}=$ 0.034. En la Figura 1 se puede apreciar que los tres tipos de interés situacional fueron predictores positivos del compromiso y el rendimiento, y predijeron negativamente el desapego; el compromiso (positivamente) y el desapego (negativamente) predijeron el rendimiento. Los efectos directos sobre el rendimiento fueron significativos solo para el interés mantenido-valor; en cambio, esos efectos directos sobre el rendimiento no fueron significativos para el interés activado ni para el interés mantenido-afecto. 
Finalmente (fase 4), se analizaron los efectos indirectos de los tipos de interés sobre el rendimiento, mediados a través del compromiso conductual y el desapego (ver Tabla 2).

La suma de los efectos indirectos fue significativa para el interés situacional activado, el mantenido-afecto y el mantenido-valor; ninguno de los intervalos de confianza incluyó el cero. Al introducir los mediadores en el modelo, el efecto directo del interés situacional activado sobre el rendimiento disminuyó drásticamente; algo análogo ocurrió con el interés situacional mantenido-afecto; en ambos casos, al incluir los mediadores en el modelo, los efectos directos dejaron de ser significativos, lo que indicaría una mediación total. En cambio, aunque la puntuación inicial total del interés situacional mantenido-valor experimentó un fuerte descenso al incluir los mediadores, sin embargo este efecto directo sigue siendo significativo, lo que está indicando una mediación parcial. Conjuntamente, las variables evaluadas explicaron un $47 \%$ de la varianza del rendimiento académico.

\section{Discusión}

El presente estudio intentó profundizar en las relaciones entre interés situacional, compromiso, desapego y rendimiento en la asignatura de Lengua Española en una muestra de estudiantes de educación secundaria obligatoria. Como paso previo, se analizaron las características de las escalas aplicadas, revelando adecuados niveles de fiabilidad y validez.

\section{Relaciones entre variables}

En general, los datos confirmaron las relaciones hipotetizadas entre las diferentes variables. En primer lugar, aquellos estudiantes que al inicio de curso percibieron las clases de Lengua como más interesante (porque el profesor despertaba su curiosidad, porque les gustaba lo que aprendían en esas clases o porque las consideraban importantes) obtuvieron al final de curso mejores notas en esa materia. Estos resultados están en la línea de investigaciones previas (Durik \& Harackiewicz, 2007; Gutiérrez \& López, 2012; Hulleman et al., 2010; Zhu et al., 2009). En este estudio, resulta llamativa la capacidad predictiva del interés situacional mantenidovalor, superior a la de los otros dos tipos de interés evaluados. Como posible razón de este resultado, se apunta la distancia entre las evaluaciones (7-8 meses), lo que pudo haber contribuido a atenuar los efectos del componente más afectivo (llamada de atención, disfrute) que está presente tanto en el interés activado como en el mantenido-afecto. En esta misma dirección apuntan Hidi et al. (2004) y Hulleman et al. (2010) cuando resaltan el papel decisivo del componente de valor, pues su efecto es más duradero que el del componente afectivo.

Los tres tipos de interés situacional también predijeron positivamente el compromiso y negativamente el desapego: aquellos estudiantes que al inicio de curso percibieron las clases de Lengua como más interesantes se comprometieron más con las tareas de clase y realizaron menos conductas indicadoras de desapego. También este hallazgo es coherente con los de investigaciones previas

TABLA 2

Efectos de cada modalidad de interés situacional sobre el rendimiento

\begin{tabular}{lcccc}
\hline \multicolumn{1}{c}{$\begin{array}{c}\text { Modalidad de } \\
\text { Interés situacional }\end{array}$} & Efectos totales & \multicolumn{2}{c}{ Efectos } & \multicolumn{2}{c}{ Efectos indirectos $\left(^{(1)}\right.$} \\
directos $(p)$ & Suma $(p)$ & Intervalo \\
\hline Activado & 0.162 & $0.08(0.109)$ & $0.082(0.01)$ & $0.024,0.152$ \\
Mantenido-Afecto & 0.219 & $0.078(0.156)$ & $0.141(0.006)$ & $0.066,0.224$ \\
Mantenido-Valor & 0.364 & $0.178(0.002)$ & $0.186(0.003)$ & $0.121,0.27$ \\
\hline
\end{tabular}

(1) La probabilidad asociada a cada suma de efectos indirectos estandarizados y su respectivo intervalo de confianza se estimaron mediante el bias-corrected confidence interval bootstrap test de AMOS.7 (intervalo de confianza = 95\%; muestras $=500$ ).

Fuente: elaboración propia. 
(Jang, 2008; Rotgans \& Schmidt, 2011; Yeung et al., 2011). Los resultados del presente estudio indican que las distintas formas de interés situacional representan un método eficaz para incrementar las conductas de implicación y compromiso con las actividades realizadas en clase y para reducir aquellas otras que denotan burnout, desapego, indiferencia y apatía. De nuevo, resulta especialmente llamativo el papel desempeñado por el interés situacional mantenido-valor: además de favorecer intensamente las conductas de compromiso, se mostró como el más eficaz protector frente a comportamientos de desapego que suelen desembocar en fracaso y abandono del colegio.

Como era de esperar, aquellos alumnos que más se comprometieron en la realización de sus tareas y que evidenciaron menos síntomas de conductas de desapego lograron mejores resultados académicos en la asignatura de Lengua. Estudios previos, con alumnos de secundaria y de universidad, habían asociado positivamente el compromiso al rendimiento (Ainley \& Ainley, 2011; Durik \& Harackiewicz, 2007; Greene et al., 2004; Jang, 2008) y a variables que lo potencian (Extremera et al., 2007: Salanova et al., 2005); en cambio, el burnout y el desapego se asociaron al fracaso escolar y al absentismo del centro (Caballero et al., 2007; Caballero et al., 2010; García et al., 2012; Martínez-Arias et al., 2007).

En línea con el modelo 3P de Biggs (Biggs et al., 2001; Justicia et al., 2008; Rosário et al., 2005), compromiso y desapego conductuales mediaron (parcial o totalmente) los efectos de las diferentes formas de interés situacional sobre el rendimiento. Una gran parte de la intensa asociación positiva entre las distintas modalidades de interés situacional y el rendimiento se explica por las relaciones expuestas anteriormente: los alumnos que perciben el entorno de su clase de Lengua como más interesante se comprometen más en las actividades académicas y muestran menos signos de desapego; ambos comportamientos (elevado compromiso y bajo desapego) anticipan mejores resultados académicos.

Conjuntamente, un porcentaje importante del rendimiento se explicó por las variables evaluadas, lo que apoya su relevancia a la hora de explicar, predecir y modificar el aprovechamiento académico de los adolescentes en esta etapa crucial de escolarización obligatoria.

\section{Implicaciones educativas}

Los resultados del presente estudio ponen de relieve el papel decisivo de los profesores para potenciar el interés situacional en clase. Para despertar el interés de los alumnos en sus clases, Hulleman et al. (2010) y Renninger (2009) recomiendan escucharles para descubrir sus preferencias e intereses; siempre que sea posible, seleccionar contenidos atractivos y relacionarlos con sucesos conocidos; establecer objetivos claros, lo mismo que el modo de evaluación y las consecuencias de la misma y utilizar materiales diversos, además de los libros.

Para mantener el interés de los estudiantes, estos autores recomiendan ayudarles a comprender la importancia de lo que estudian; favorecer la participación activa y la interacción social con los compañeros; proponerles múltiples tareas, prácticas a ser posible, además de las exposiciones magistrales y, de forma ocasional, realizar algo sorprendente, distinto de lo que esperan que ocurra. También resulta eficaz que el profesor manifieste su interés por los contenidos que imparte, proyectando entusiasmo en lo que enseña, introduciendo las tareas de forma positiva, destacando la utilidad del tema y esperando el interés de sus alumnos.

Otros autores proponen a los docentes diferentes estrategias que contribuyen a mejorar el compromiso en las aulas y a reducir el desapego y el abandono (Jang, 2008; Martin, 2008; Pianta, Hamre \& Allen, 2012). Algunas de esas propuestas son: ponerse en el lugar del alumno e intentar comprender su perspectiva; descubrir las fortalezas y debilidades de cada estudiante y el modo de mejorar sus resultados; plantearles actividades que representen para ellos un reto óptimo y ofrecerles razones para realizarlas; utilizar lo menos posible un lenguaje controlador; reconocer y aceptar expresiones de afecto negativo por parte de los alumnos; presentarles normas e instrucciones claras y comprensibles y proporcionarles 
feedback constructivo sobre el modo en que pueden ejercer el control sobre sus resultados académicos.

Ambas líneas de intervención, para potenciar el interés y para mejorar el compromiso reduciendo el desapego, entroncan con diferentes modelos que consideran la autorregulación como un proceso interactivo de enseñanza y aprendizaje (Cleary \& Zimmerman, 2012; De la Fuente \& Justicia, 2007; Pianta et al., 2012; Wolters \& Taylor, 2012). En ellos se explicitan determinadas características de la enseñanza que potencian en el alumno la autorregulación de su aprendizaje.

\section{Limitaciones y futuras investigaciones}

Este trabajo analizó, como predictor, el interés situacional. Otras variables presagio relevantes podrían ser los enfoques de aprendizaje (Biggs et al., 2001; Justicia et al., 2008) o diferentes estrategias autorreguladoras (De la Fuente \& Justicia, 2007).

Además, este estudio abordó solo el componente conductual del compromiso y el desapego. En investigaciones futuras, estos resultados podrían completarse evaluando los demás componentes clásicos (Fredricks et al., 2004): el cognitivo, como la utilización de estrategias y autorregulación del aprendizaje (Cleary \& Zimmerman, 2012; De la Fuente \& Justicia, 2007; Wolters \& Taylor, 2012) y el emocional, que se concreta en reacciones afectivas ante las tareas de clase, como el disfrute o el estrés académico (Barraza, 2008; Extremera et al., 2007; Feldman et al., 2008).

Para la evaluación del producto final del aprendizaje se utilizó el rendimiento académico. Para complementar esta perspectiva, en el futuro podrían evaluarse otros indicadores, como la calidad de los procesos de aprendizaje (Rosário et al., 2005) o el grado en que se han alcanzado determinadas competencias (Solanes, Núñez \& Rodríguez, 2008).

Aunque costosos y no exentos de dificultades, sería de gran utilidad llevar a cabo estudios longitudinales durante varios cursos de secundaria para conocer las relaciones cíclicas y recíprocas entre las variables evaluadas y el nacimiento del interés personal; ese análisis sería especialmente esclarecedor en materias que se incorporan por primera vez al currículum académico en esta etapa educativa, como son tecnología, física, química, geología o biología; esto permitiría averiguar de qué modo y en qué medida el comportamiento de los profesores (percibido por sus alumnos) condiciona en estos el nacimiento de intereses personales duraderos hacia esas materias nuevas.

\section{Referencias}

Ainley, M. (2012). Students' interest and engagement in classroom activities. En S. Christenson, A. Reschly \& C. Wylie (Eds.), Handbook of research on student engagement (pp. 283-302). Nueva York: Springer.

Ainley, M. \& Ainley, J. (2011). Student engagement with science in early adolescence: The contribution of enjoyment to students' continuing interest in learning about science. Contemporary Educational Psychology, 36(1), 4-12.

Alonso Tapia, J. (2007). Evaluación de la motivación en entornos educativos. En M. Álvarez \& R. Bisquerra (Eds.), Manual de orientación y tutoría (pp. 1-37). Barcelona: Kluwer.

Arbuckle, J. (2006). AMOS 7.0. User's Guide. Chicago, IL: Smallwaters Corporation.

Barraza, A. (2008). El estrés académico en alumnos de maestría y sus variables moduladoras: un diseño de diferencias de grupos. Avances en Psicología Latinoamericana, 26(2), 270-289.

Biggs, J., Kembler, D. \& Leung, D. (2001). The revised two-factor Study Process Questionnaire: R-SPQ2F. British Journal of Educational Psychology, 71(1), 133-149.

Brislin, R. (1986). The wording and translation of research instruments. En W. Lonner \& J. Berry (Eds.), Field methods in cross-cultural research (pp. 137-164). Beverly Hills, CA: Sage.

Byrne, B. (2010). Structural equation modeling with AMOS. Basic concepts, applications, and programming (2a. ed.). New York: Routledge.

Caballero, C., Abello, R. \& Palacio, J. (2007). Relación del burnout y el rendimiento académico con la satisfacción frente a los estudios en estudiantes universitarios. Avances en Psicología Latinoamericana, 25(2), 98-111. 
Caballero, C., Hederich, C. \& Palacio, J. (2010). El burnout académico: delimitación del síndrome y factores asociados con su aparición. Revista Latinoamericana de Psicología, 42(1), 131-146.

Caso-Niebla, J. \& Hernández-Guzmán, L. (2007). Variables que inciden en el rendimiento académico de adolescentes mexicanos. Revista Latinoamericana de Psicología, 39(3), 487-501.

Cleary, T. \& Zimmerman, B. (2012). A cyclical self-regulatory account of student engagement: Theoretical foundations and applications. En S. Christenson, A. Reschly \& C. Wylie (Eds.), Handbook of research on student engagement (pp. 237-257). New York: Springer.

De la Fuente, J. \& Justicia, F. (2007). El modelo DIDE$\mathrm{PRO} \circledast$ de regulación de la enseñanza y del aprendizaje: avances recientes. Education \& Psychology, 5(13), 535-564.

Durik, A. \& Harackiewicz, J. (2007). Different strokes for diferent folks: How individual interest moderates the effects of situational factors on task interest. Journal of Educational Psychology, 99(3), 597-610.

Extremera, N., Durán, A. \& Rey, L. (2007). Inteligencia emocional y su relación con los niveles de burnout, engagement y estrés en estudiantes universitarios. Revista de Educación, 342, 239-256.

Feldman, L., Goncalves, L., Chacón-Piugnau, G., Zaragoza, J., Bagés, N. \& de Pablo, J. (2008). Relaciones entre estrés académico, apoyo social, salud mental y rendimiento académico en estudiantes universitarios venezolanos. Universitas Psychologica, 7(3), 739-751.

Fredricks, J., Blumenfeld, P. \& Paris, A. (2004). School engagement: Potential of the concept, state of the evidence. Review of Educational Research, 74(1), 59-109.

García, M., Casal, J., Merino, R. \& Sánchez, A. (2012). Itinerarios de abandono escolar y transiciones tras la enseñanza secundaria obligatoria. Revista de Educación, 361, 1-15.

García-Señorán, M. \& González González, S. (2008). Interés, capacidad percibida y rendimiento académico en alumnos de Educación Secundaria Obligatoria. Informes Psicológicos, 10(11), 207-224.

González González, M. (2010). El alumno ante la escuela y su propio aprendizaje: algunas líneas de investiga- ción en torno al concepto de implicación. Revista Iberoamericana sobre Calidad, Eficacia y Cambio en Educación, 8(4), 11-31.

Greene, B., Miller, R., Crowson, M., Duke, B. \& Akey, K. (2004). Predicting high school students' cognitive engagement and achievement: Contributions of classroom perceptions and motivation. Contemporary Educational Psychology, 29(4), 462-482.

Gutiérrez, M. \& López, E. (2012). Motivación, comportamiento de los alumnos y rendimiento académico. Infancia y Aprendizaje, 35(1), 61-72.

Hidi, S. \& Renninger, K. (2006). The four-phase model of interest development. Educational Psychologist, 41(2), 111-127.

Hidi, S., Renninger, K. \& Krapp, A. (2004). Interest, a motivational variable that combines affective and cognitive functioning. En D. Dai \& R. Sternberg (Eds.), Motivation, emotion, and cognition. Integrative perspectives on intellectual functioning and development (pp. 89-115). Mahwah, NJ: LEA.

Hu, L. \& Bentler, P. (1999). Cutoff criteria for fit indexes in covariance structure analysis: Conventional criteria versus alternatives. Structural Equation Modeling, 6(1), 1-55.

Hulleman, C., Godes, O., Hendricks, B. \& Harackiewicz, J. (2010). Enhancing interest and performance with a utility value intervention. Journal of Educational Psychology, 102(4), 880-895.

Jang, H. (2008). Supporting students' motivation, engagement, and learning during an uninteresting activity. Journal of Educational Psychology, 100(4), 798-811.

Justicia, F., Pichardo, M., Cano, F., Berbén, A. \& De la Fuente, J. (2008). The revised two-factor Study Process Questionnaire (R-SPQ-2F): Exploratory and confirmatory factor analyses at item level. European Journal of Psychology of Education, 23(3), 355-372.

Krapp, A. \& Prenzel, M. (2011). Research on interest in science: Theories, methods, and findings. International Journal of Science Education, 33(1), 27-50. Linnenbrink-García, L., Durik, A., Conley, A., Barron, K., Tauer, J., Karabenick, S., et al. (2010). Measuring situational interest in academic domains. Educational and Psychological Measurement, 70(4), 647-671. 
Macías-Martínez, D. \& Hernández-Pozo, M. (2008). Indicadores conductuales de ansiedad escolar en bachilleres en función de sus calificaciones en un examen de matemáticas. Universitas Psychologica, $7(3), 767-785$.

Manzano, G. (2004). Perfil de los estudiantes comprometidos con sus estudios: influencia del burnout y el engagement. Anuario de Psicología, 35(3), 399. 415.

Martin, A. (2008). Enhancing student motivation and engagement: The effects of a multidimensional intervention. Contemporary Educational Psychology, 33(2), 239-269.

Martin, A. \& Liem, G. (2010). Academic personal bests (PBs), engagement, and achievement: $A$ cross-lagged panel analysis. Learning and Individual Differences, 20(3), 265-270.

Martínez-Arias, R., Aguado, P., Álvarez, M., Colodrón, M. \& Gallego, E. (2007). Prevalencia del absentismo escolar en la Educación Secundaria Obligatoria: relación con variables sociodemográficas y familiares. Psicología Educativa, 13(1), 35-63.

Ministerio de Educación (2010). PISA 2009. Programa para la Evaluación Internacional de los Alumnos OCDE. Informe Español. Madrid: Autor.

Miñano, P., Gilar, R. \& Castejón, J. L. (2012). A structural model of cognitive-motivational variables as explanatory factors of academic achievement in Spanish language and mathematics. Anales de Psicología, 28(1), 45-54.

Núñez, J., Martín-Albo, J., Paredes, A., Rodriguez, O. \& Chipana, N. (2011). The mediating role of perceived competence: Testing a motivational sequence in university students. Universitas Psychologica, 10(3), 669-680.

Parra, P. (2010). Relación entre el nivel de engagement y el rendimiento académico teórico/práctico. Revista de Educación en Ciencias de la Salud, 7(1), 57-63.

Pérez, M., Valenzuela, M., Díaz, A., González-Pienda, J. \& Núñez, J. (2010). Disposición y enfoques de aprendizaje en estudiantes universitarios de primer año. Universitas Psychologica, 10(2), 441-449.

Pianta, R., Hamre, B. \& Allen, J. (2012). Teacherstudent relationships and engagement: Conceptualizing, measuring, and improving the capacity of classroom interactions. En S. Christenson, A.
Reschly \& C. Wylie (Eds.), Handbook of research on student engagement (pp. 365-386). New York: Springer.

Renninger, K. A. (2009). Interest and identity development in instruction: An inductive model. Educational Psychologist, 44(2), 105-118.

Ros, I. (2009). La implicación del estudiante con la escuela. Revista de Psicodidáctica, 14(1), 79-92.

Rosário, P., Núñez, J., González-Pienda, J., Almeida, L., Soares, S. \& Rubio, M. (2005). El aprendizaje escolar examinado desde la perspectiva del "Modelo 3P" de J. Biggs. Psicothema, 17(1), 20-30.

Rotgans, J. \& Schmidt, H. (2011). Situational interest and achievement in the active-learning classroom. Learning and Instruction, 21(1), 58-67.

Salanova, M., Martínez, I., Bresó, E., Llorens, S. \& Grau, R. (2005). Bienestar psicológico en estudiantes universitarios: facilitadores y obstaculizadores del desempeño académico. Anales de Psicología, 21(1), 170-180.

Schiefele, U. (2009). Situational and individual interest. En K. Wentzel \& A. Wigfield (Eds.), Handbook of motivation at school (pp. 197-222). Londres: Routledge.

Skinner, E., Furrer, C., Marchand, G. \& Kinderman, T. (2008). Engagement and disaffection in the classroom: Part of a larger motivational dynamic? Journal of Educational Psychology, 100(4), 765-781.

Skinner, E., Kinderman, T., Connell, J. \& Wellborn, J. (2009). Engagement and disaffection as organizational constructs in the dynamic of motivational development. En K. Wentzel \& A. Wigfield (Eds.), Handbook of motivation at school (pp. 223-245). Londres: Routledge.

Skinner, E., Kinderman, T. \& Furrer, C. (2009). A motivational perspective on engagement and disaffection. Conceptualization and assessment of children's behavioral and emotional participation in the academic activities in the classroom. Educational and Psychological Measurement, 69(3), 493-525.

Skinner, E. \& Pitzer, J. (2012). Developmental dynamics of student engagement, doping, and everyday resilience. En S. Christenson, A. Reschly \& C. Wylie (Eds.), Handbook of research on student engagement (pp. 21-44). Nueva York: Springer. 
Solanes, A., Núñez, R. \& Rodríguez, J (2008). Elaboración de un cuestionario para la valuación de las competencias genéricas en estudiantes universitarios. Apuntes de Psicología, 26(1), 35-49.

Valle, A., Rodríguez, S., Cabanach, R., Núñez, J., González, J. \& Rosario, P. (2010). Perfiles motivacionales y diferencias en variables afectivas, motivacionales y de logro. Universitas Psychologica, 9(1), 109-121.

Wang, M. \& Holcombe, R. (2010). Adolescents' perceptions of school environment, engagement, and academic achievement in middle school. American Educational Research Journal, 47(3), 633-662.

Wolters, C. \& Taylor, D. (2012). A self-regulating learning perspective on student engagement. En S. Christenson, A. Reschly \& C. Wylie (Eds.), Han- dbook of research on student engagement (pp. 635 651). New York: Springer.

Wu, A. \& Zumbo, B. (2008). Understanding and using mediators and moderators. Social Indicators Research, 87(3), 367-392.

Yeung, A., Lau, S. \& Nie, Y. (2011). Primary and secondary students' motivation in learning English: Grade and gender differences. Contemporary Educational Psychology, 36(3), 246-256.

Zhu, X., Chen, A., Ennis, C., Sun, H., Hopple, C., Bonello, M., et al. (2009). Situational interest, cognitive engagement, and achievement in physical education. Contemporary Educational Psychology, 34(3), 221-229. 
